\title{
Neuropsychiatric changes in Parkinson's disease patients: A prospective observational two years study
}

\author{
Dan Iulian Cuciureanu' ${ }^{1,2}$, Cristina-Georgiana Croitoru ${ }^{1,2}$, Victor Constantinescu', \\ Liviu Bolohan'2, Tudor Cuciureanu ${ }^{1,3}$ \\ 1"Grigore T. Popa" University of Medicine and Pharmacy, lasi, Romania \\ 2"Prof. Dr. N. Oblu" Emergency Clinical Hospital, lasi, Romania \\ ${ }^{3}$ Institute of Gastroenterology and Hepatology, Iasi, Romania
}

\begin{abstract}
Parkinson's disease (PD) is clinically manifested by motor and non-motor symptoms, including neuropsychiatric changes, which can occur both in the early and advanced stages of PD.

Objectives. The study evaluated cognitive and behavioral changes in patients with PD.

Material and methods. Unified Parkinson's Disease Rating Scale (UPDRS) and Hoehn and Yahr scale were used to assess the neurological status of 112 patients with PD. Clinical neuropsychological tests were applied to all patients.

Outcomes. The longer the duration of the disease is, the more critical impulse-control disorders are, especially shopping, hobbyism, and punding. Hypersexual behavior appears to be age and gender-dependent. Depression and impulse-control disorders seem interconnected: subjects tested with severe depression on the Hamilton Depression Rating Scale have more shopping compulsions. Antiparkinsonian treatment influences the Montreal Cognitive Assessment score. When specific cognitive domains are tested, subjects treated only with levodopa have lower scores at temporal and spatial orientation testing. Dopamine agonists appear to be associated with less cognitive dysfunctions.

Conclusions. Neuropsychiatric symptoms impact the quality of life in Parkinson's disease patients and must be systematically assessed.
\end{abstract}

Keywords: Parkinson's disease, depression, cognitive dysfunction

\section{INTRODUCTION}

Parkinson's disease (PD), first described as a neurological syndrome by James Parkinson in 1817 (1), has been a central piece of interest among the medical communities worldwide. Today sporadic $\mathrm{PD}$ is the second most frequent neurodegenerative disorder after Alzheimer's disease (2). According to the International Parkinson and Movement Disorders Society (MDS) Task Force, early PD is divided into preclinical, prodromal, and clinical phases (3). The prodromal PD is defined as a cumulus of neurodegeneration signs and symptoms which do not exceed the threshold for clinical PD
(4). The probability that patients in the neurodegenerative state may develop PD is estimated using prodromal markers as abnormal dopaminergic PET/SPECT, olfactory loss, constipation, anxiety or depression, and several risk markers such as male sex, non-use of caffeine and smoking (5).

Braak's theory represents the basis of the concept that sporadic PD is a multisystemic disorder according to which neurodegeneration progressively spreads throughout responsive limbic, autonomic, and somatomotor sites of the nervous system in a six-stage pattern (6). According to this theory, the first affected sites are the olfactory bulb, anterior part of the olfactory nucleus, preganglionic para- 
sympathetic neurons of the dorsal vagal motor nucleus and enteric nervous system of the foregut (7). In the advanced stages of sporadic PD, neurodegeneration affects striatum, anteromedial temporal mesocortex and key components of the neocortex such as premotor fields and sensory association areas $(6,7)$.

Even though motor features have been most studied, recent data emphasizes the importance of non-motor features, mainly due to their increased prevalence and impact on the quality of life. The main non-motor symptoms which can occur both in the early and advanced stages of PD include not only sensorial and autonomic ones but also cognitive and neuropsychiatric features (8). Most of these behavioral changes and cognitive disturbances are underdiagnosed and therefore, untreated in the early stages of PD. Patients with hyperdopaminergic state due to dopamine replacement therapy tend to be affected by another range of neuropsychiatric disorders, such as impulse control disorder, hallucinations, and psychosis $(9,10)$.

This observational prospective epidemiological study aims to assess neuropsychiatric disorders present in patients with PD from North-Eastern part of our country.

\section{MATERIAL AND METHODS}

112 patients with PD were recruited from the neurological department within 24 months. All patients were duly informed according to the study protocol and consented to the assessment following ethical principles. The study was carried out in accordance with the Helsinki Declaration.

For each patient, the new MDS diagnostic criteria for PD were applied. Patients with less than eight school grades were excluded in order to avoid possible false results at cognitive testing due to lack of task understanding. Patients that presented a cerebral event in the last six months, which could alter cognitive assessment were also excluded.

Each patient was assessed by an experienced neurologist that used motor Unified Parkinson's Disease Rating Scale (UPDRS). A specialized clinical psychologist applied clinical neuropsychological tests to all subjects. These standardized tests included Montreal Cognitive Assessment (MoCA),
Hamilton Depression Rating Scale (HAM-D), Questionnaire for Impulsive-Compulsive Disorders in Parkinson's Disease Rating Scale (QUIPRS) and Global Assessment of Functioning Scale (GAF).

MoCA is a standardized screening tool for cognitive testing (11). It evaluates eight cognitive domains using a 30-point test that can be rapidly performed (12). In our study, the clinical psychologist focused on five relevant cognitive factors: executive function, language, attention, memory, and temporal and spatial orientation.

HAM-D is one of the most frequently used scales to evaluate depression. The patients were assessed using the standard seventeen item version of HAM-D (13).

QUIP-RS is a standardized instrument meant to quantify the severity of impulsive-compulsive syndromes and related disorders such as hobbysm, punding, and dopamine dysregulation syndrome in patients diagnosed with PD. It is derived from the Questionnaire for Impulsive-Compulsive Disorders in Parkinson's Disease, which is just a screening tool. QUIP-RS appraises each symptom by its frequency (14).

GAF scale is used to globally assess the functioning of an individual in terms of psychological, social, and occupational integration (15).

The statistical analysis was performed using SPSS 17.0. Scores obtained at the neuropsychiatric tests were considered the dependent variables. P values $<0.05$ were considered significant.

\section{OUTCOMES}

We evaluated 62 male and 50 female patients with PD, with a median age of 66 years. Three major categories were considered: young adults (age $<50$-year-old), adults (age between 50 and 65-yearold ) and elderly ( $>65$-year-old), the last group comprising $50.4 \%$ of cases. Regarding age at onset of the symptoms, three categories emerged: onset at less than 56-year-old (33\% of cases), age between 56 and 66 -year-old (34\% of cases) and late-onset, after 66 -year-old (33\% of cases). The mean age of onset was 61 years. The duration of the disease in the analyzed patients is illustrated in figure 1 .

Data referring to antiparkinsonian treatment revealed that $61.9 \%$ of patients were taking both lev- 
odopa and dopamine agonists, whereas $22.1 \%$ underwent dopamine agonist treatment, and 9.7\% were treated only with levodopa.

When subjects performed MoCA testing, none of them obtained results suggestive for severe cognitive impairment. $10.6 \%$ of patients had mild cognitive impairment, $38.4 \%$ had a normal cognitive function, while the majority of $50.9 \%$ had some degree of cognitive dysfunction. The average GAF score was 49.5 and only $16.1 \%$ of patients obtained 31 points or less. $29.5 \%$ of the subjects obtained a total score of 0 at QUIP-RS. Hobbysm and pundinghad the highest scores of 1.6, respectively 1.34 . The frequency of impulsive-compulsive symptoms is illustrated in figure 1 .
Patients' gender influences HAM-D score $(\mathrm{p}=$ 0.020): females obtained a higher score (15.86) than men (12.66). Also, there is a significant difference between gender and sexual compulsions $(\mathrm{p}=0.042)$ on QUIP-RS, with a higher score in men.

Patients' age appears to influence overall cognitive function $(\mathrm{p}=0.028)$, GAF score $(\mathrm{p}=0.034)$, and sexual compulsions. The highest MoCA scores are obtained in the adults' category $(\mathrm{M}=25.13)$, whereas significantly lower values are registered in the elderly group $(M=23.17)$, especially in the memory $(\mathrm{p}=0.034)$ and executive function $(\mathrm{p}=$ 0.010 ) domains (figure 2). The highest GAF scores are obtained by subjects in the adult category $(\mathrm{M}=$ 52.95), followed by the elderly $(M=46.6)$, while

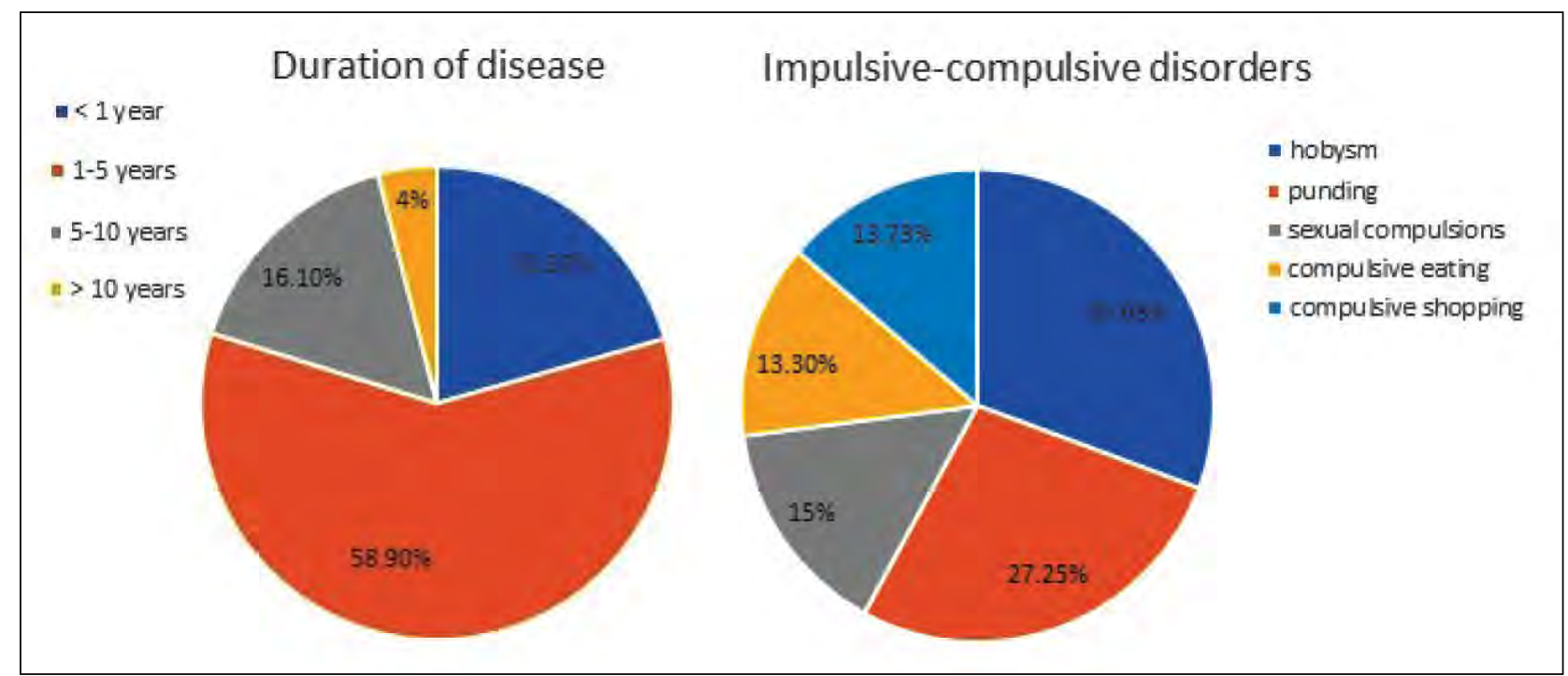

FIGURE 1. Duration of disease and characteristics of the impulsive-compulsive disorders
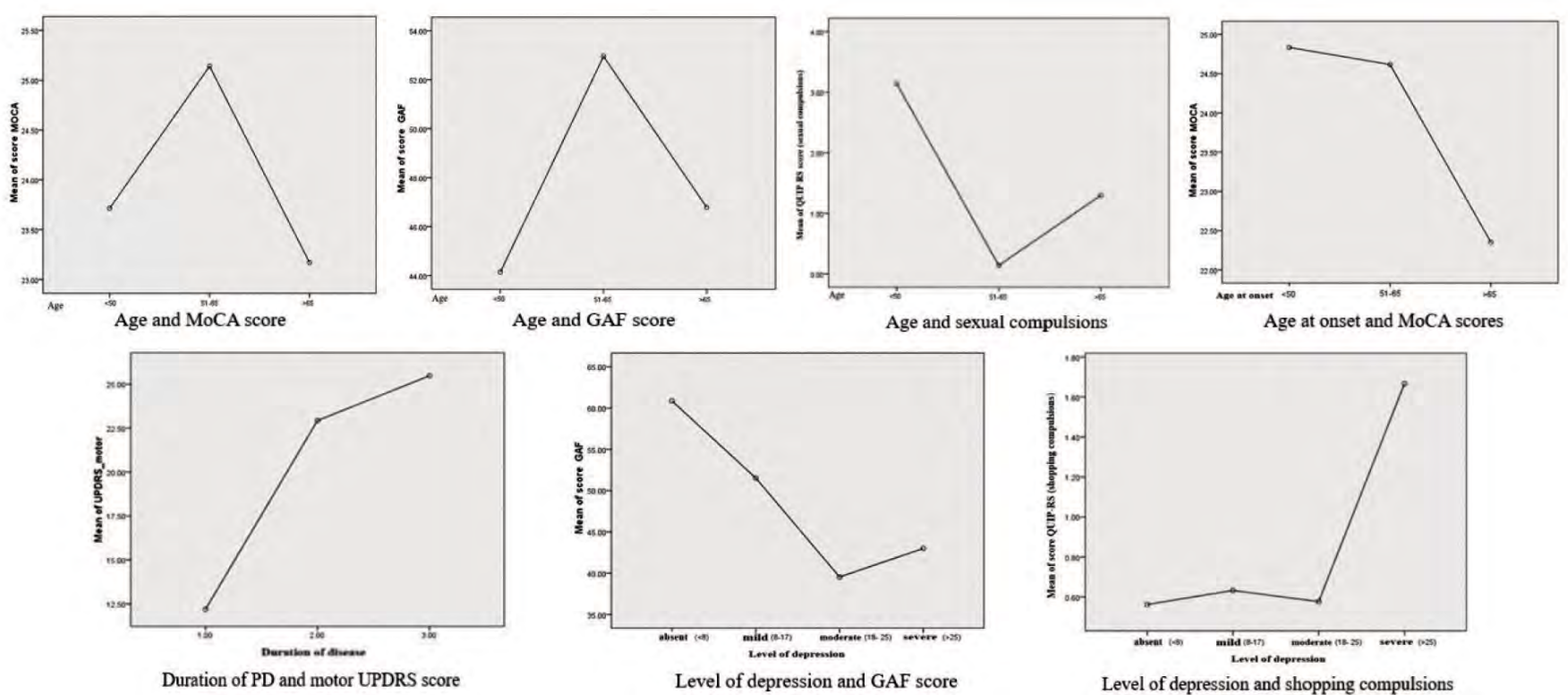

Level of depression and GAF score

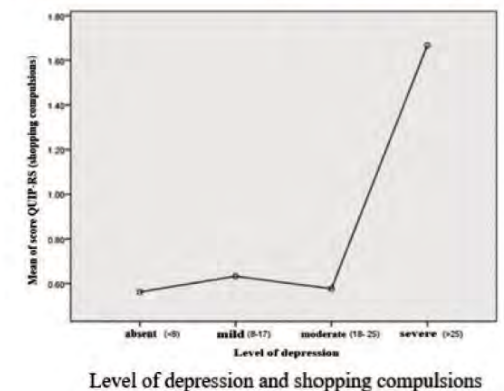

Level of depression and shopping compulsions

FIGURE 2. Clinical features and measurements in PD patients 
the lowest scores were registered in the young adult category $(M=40.66)$ (figure 2$)$. Regarding age and sexual compulsions, the highest score was obtained within the young adult's category $(\mathrm{M}=3.14)$ compared with adults $(\mathrm{M}=0.13)$ and elderly $(\mathrm{M}=1.29)$ $(\mathrm{p}=0.002)$ (figure 2).

Age at onset influences MoCA score $(p=0.004)$. As shown in figure 2, the higher the age at onset, the lower the MoCA scores are. From all eight cognitive domains included, executive function is most influenced $(p=0.020)$. GAF values closely vary with age at onset, the lowest values being observed in patients in which PD was clinically obvious after 66 years $(p=0.009)$. Also, in this category, we found lower scores in QUIP-RS compared with the category 56-66 years, with a special emphasis on compulsive eating $(\mathrm{p}=0.016)$ and compulsive shopping $(p=0.015)$. Subjects with age at onset 56 years or less obtained a lower value on motor UPDRS compared with the other two categories ( $\mathrm{p}=$ 0.036 and $p=0.038$ respectively).

The duration of PD significantly influences the QUIP-RS score $(\mathrm{p}=0.002)$ and motor UPDRS $(\mathrm{p}=$ 0.009 ) in a directly proportional manner, as shown in figure 2.

From all possible connections tested, Hoehn and Yahr stage influences GAF score inversely proportional. Level of tuition influences certain cognitive domains such as language, in a directly proportional manner $(p=0.018)$ and attention. Subjects with higher education have better results in the part of MoCA dedicated to attention than subjects with secondary education.

When variable antiparkinsonian treatment was studied, it appeared to influenceMoCA score. A combined levodopa and dopamine agonist treatment is associated with a higher MoCA score, whereas isolated levodopa treatment is associated with the lowest score $(p=0.013)$. When specific cognitive domains are tested, again, subjects treated only with levodopa have lower scores at temporal and spatial orientation testing.

The severity of depression estimated with the HAM-D scale influenced inversely proportional the GAF scores $(\mathrm{p}<0.001)$ (figure 2). Post hoc tests revealed that patients without depression obtained higher MoCA scores compared with patients with mild $(\mathrm{p}=0.033)$ and moderate depression $(\mathrm{p}=$ 0.014). Moderate depression was associated with lower attention $(p=0.011)$ and executive function $(p=0.09)$. Severe depression is correlated with elevated shopping compulsions (figure 2).

A higher male/female ratio was systematically reported (16), similar to our findings. However, the risk of developing PD for women increases with age $(16,17)$. This is probably due to a cumulus of exogenous and endogenous factors such as lifestyle exposures, genetic effects, and the most disputed neuroprotective role of estrogen hormones on the main mesostriatal dopaminergic pathway (16).

The majority of subjects diagnosed with PD in our study were older than 65 years, according to the data from literature (18).

Approximately $11 \%$ of patients included in our study had mild cognitive impairment. The percentage is significantly lower than the average of $25 \%$ obtained in other epidemiological studies (19). The difference in mild cognitive impairment's frequency may be explained by the different tools used to screen this condition: in the present study, we used MoCA whereas other standardized neuropsychological tests such as Dementia Rating Scale and Parkinson's Disease Cognitive Rating Scale were used in the publication mentioned above.

The present study reveals that as the subjects' age increases over 65 years, there is a significant decrease in cognitive function, especially in memory and executive function. The severity of depression negatively influences cognitive functions, the lowest MoCA scores being obtained in patients with mild and moderate depression. All these results place increased age at assessment, depression, and advanced age of onset in the pool of potential risk factors for cognitive decline as stated in the medical literature $(20,21)$.

The association between levodopa and dopamine agonist offers mid-term protection against cognitive impairment compared to isolated levodopa treatment. These differences may reside in the two different types of dopaminergic stimulation, phasic by levodopa and tonic by dopamine agonists $(22,23)$. Even though acute dopaminergic stimulation with dopamine agonists has a beneficial effect on specific cognitive domains such as working memory, few data are available regarding chronic cognitive effects of levodopa versus dopamine agonists in prolonged therapy (24).

Almost half of the subjects included in the present study have a moderate overall functional im- 
pairment defined by an average GAF score of 49.5. Less than one-fifth of the cases experience a significant limitation of activities of daily living. As expected, the more advanced the Hoehn and Yahr stage of PD is, the lower the GAF score becomes. Also, patients with older age at onset have worse global functionality than patients with age at onset lower than 66 years. This can be explained by the known fact that older age at onset is associated with much more important dopamine dysfunctions and, therefore, with much more invalidating motor and non-motor symptoms (25). Older age at onset represents a predictive factor for more rapid motor aggravation and nursing home placement (26). However, surprisingly, patients younger than 50 years appear to have the most significant overall functional impairment. A possible justification may reside in the longer duration of dopaminergic treatment, which is correlated with more severe motor and non-motor complications (17). When the level of depression was taken into consideration, we observed that the higher the HAM-D score is, the lower the GAF score becomes. One can deduce that depression has a more considerable influence on global functionality than the severity or duration of the disease.

Neuropsychiatric symptoms are the most frequent non-motor feature in patients with untreated early stages of PD (27). Depression, sleep disturbance, anxiety, and apathy were the most encountered, especially in association with more severe motor symptoms. On the opposite side of the spectrum, psychotic symptoms were scarce among treatment naïve patients (27). Even though most of the early neuropsychiatric signs are overlooked, it is of critical importance to assess these prodromal PD changes, as one category can be a predictor for the other. For example, an early executive function deficit may be a reliable predictor for apathy in the future course of the disease (28).

From neuropsychiatric disorders associated with PD, impulse-control disorders are a heterogeneous group of compulsive and repetitive manifestations, most frequently represented by pathological gambling, compulsive shopping, hypersexuality, compulsive eating and followed by punding and hoarding (29). Almost one-third of the subjects have impulse-control disorders, mostly hobbyism and punding.
Unlike other behavioral changes that appear to be independent of age at onset, impulse-control disorders appear to be correlated with early-onset PD among other risk factors, such as male gender, previous history of drug abuse, excessive alcohol consumption, or smoking $(29,30)$. In the current study, patients with age at onset between 55 to 66 years, experience more frequently impulsions and compulsions, mostly regarding binge eating and compulsive shopping. No cause-effect relation was found in the present study between these specific impulse-control disorders and female gender, as mentioned in the medical literature (31). The present study revealed that the longer the duration of the disease is, the more critical impulse-control disorders are, especially shopping, hobbyism, and punding.

In our study, hypersexual behavior appears to be age and gender-dependent. Depression and impulse-control disorders seem interconnected: subjects with severe depression on the HAM-D scale have more significant shopping compulsions.

The most unexpected result regarding impulse-control disorders in our study was the lack of correlation between these behavioral symptoms and dopaminergic treatment. It is a generally known fact that impulse-control disorders, especially pathological gambling, are associated with dopaminergic treatment (32). As a consequence of limbic ventral striatum hyperstimulation, impulse-control disorders are considered a side-effect of dopaminergic treatment, more frequently associated with dopamine agonists than levodopa (29). A possible explanation for this variation in this study resides in its short duration of observation.

It is generally accepted that depressive symptoms emerge earlier than motor signs of PD and can constitute a form of onset (33). On one side, depression can influence cognitive functions, global functionality, or types of compulsions in patients diagnosed with PD, as shown in the present study. On the other hand, depression in patients with PD is influenced by several factors, such as personal or family history of depression, early-onset of the disease, psychiatric comorbidities, or female gender. These characteristics are known as possible risk factors for depression in PD (20). In the current study, female patients had systematically obtained higher scores in HAM-D than men. 


\section{CONCLUSIONS}

The current study contributes to an overview of patients with PD from the North-Eastern part of our country. We did not find a correlation between treatment with dopamine agonists and impulse-control disorders in our patients. Dopamine agonists appear to be associated with less cognitive dysfunctions. Both results must be interpreted under the reserve of the short duration of the study. Given the limited period of observation of our patients, no conclusion can be drawn regarding the long-term effects of antiparkinsonian treatment. Further studies on larger groups of patients are needed. Thus, the assessment of the cognitive and neuropsychiatric features in clinical practice opens new perspectives of research for therapeutic strategy in PD patients.

\section{Acknowledgement}

Special thanks for the patients that accepted to be evaluated and included in this research.

\section{Conflict of interest: none declared} Financial support: none declared

\section{REFERENCES}

1. Parkinson J.An Essay on the Shaking Palsy. Whittingham and Rowland for Sherwood, Neely, and Jones. London; 1817.

2. de Lau LML, Breteler MMB. Epidemiology of Parkinson's disease. Lancet Neurol. 2006;5:525-535.

3. Berg D, Postuma RB, Bloem B et al. Time to redefine PD? Introductory statement of the MDS Task Force on the definition of Parkinson's disease. Mov Disord. 2014;29(4):454-462.

4. Postuma RB, Berg D, Stern M et al.MDS clinical diagnostic criteria for Parkinson's disease. Mov Disord. 2015;30(12):1591-1600.

5. Marsili L, Rizzo G, Colosimo C. Diagnostic Criteria for Parkinson's Disease: From James Parkinson to the Concept of Prodromal Disease. Front Neurol. 2018;9:156.

6. Braak H, Ghebremedhin E, Rüb Uet al.Stages in the development of Parkinson's disease-related pathology. Cell Tissue Res. 2004; 318(1):121-134.

7. Braak H, Del Tredici K. Nervous system pathology in sporadic Parkinson disease. Neurology. 2008;70(20):1916-1925.

8. Balestrino R, Martinez-Martin P. Neuropsychiatric symptoms, behavioural disorders, and quality of life in Parkinson's disease. J Neurol Sci. 2017;373:173-178.

9. Latella D, Maggio MG, Maresca $G$ et al. Impulse control disorders in Parkinson's disease: A systematic review on risk factors and pathophysiology. J Neurol Sci. 2019;398:101-106.

10. Cuciureanu DI, Cuciureanu T, Popescu RM et al. Dopaminergic centers neurodegenaration - biochemical and radiologic approach. Rev Chim (Bucharest). 2019;3(70):1835-1925.

11. Kang JM, Cho YS, Park et al. Montreal cognitive assessment reflects cognitive reserve. BMC geriatrics. 2018;18(1):261.

12. Nasreddine ZS, Phillips NA, Bédirian V et al. The Montreal cognitive assessment, MoCA: A brief screening tool for mild cognitive impairment. J Am Geriatr Soc. 2005;53(4):695-699.

13. Hamilton M. A rating scale for depression. $J$ Neurol Neurosurg Psychiatry. 1960;23(1):56-62.

14. Weintraub D, Mamikonyan E, Papay K et al. Questionnaire for Impulsive-Compulsive Disorders in Parkinson's Disease-Rating Scale. Mov Disord. 2012; 27(2):242-247.

15. Aas $\mathrm{IH}$. Guidelines for rating Global Assessment of Functioning (GAF). Ann Gen Psychiatry. 2011;10:2.

16. Moisan F, Kab S, Mohamed Fet al.Parkinson disease male-to-female ratios increase with age: French nationwide study and meta-analysis J Neurol Neurosurg Psychiatry. 2016;87:952-957.

17. DeMaagd G, Philip A. Parkinson's Disease and Its Management: Part 1: Disease Entity, Risk Factors, Pathophysiology, Clinical Presentation, and Diagnosis. P T. 2015;40(8):504-532.

18. Alves G, Forsaa EB, Pedersen KFet al.Epidemiology of Parkinson's disease, Journal of Neurology. 2008;255 (Suppl 5):18-32.
19. Aarsland D, Bronnick K, Williams-Gray $\mathrm{CH}$ et al.Mild cognitive impairment in Parkinson's disease: a multicentre pooled analysis. Neurology. 2010;75:1062-1069.

20. Weintraub D, Burn DJ. Parkinson's Disease: The Quintessential Neuropsychiatric Disorder. Mov Disord. 2011;26(6):1022-1031.

21. Xing $Y$, Tang $Y$, Zhao $L$ et al. Associations between plasma ceramides and cognitive and neuropsychiatric manifestations in Parkinson's disease dementia. J Neurol Sci. 2016;370:82-87.

22. Bonuccelli U, Pavese N. Dopamine agonists in the treatment of Parkinson's disease. Expert Rev Neurother. 2006;6:81-89.

23. Poewe $\mathrm{W}$, Antonini $\mathrm{A}$, Zijlmans $\mathrm{J}$ et al.Levodopa in the treatment of Parkinson's disease: an old drug still going strong. Clin Interv Aging. 2010;7:229-238.

24. Poletti M, Bonuccelli U. Acute and chronic cognitive effects of levodopa and dopamine agonists on patients with Parkinson's disease: A review. TherAdv Psychopharmacol. 2013;3(2):101-113.

25. Pagano G, Ferrara N, Brooks DJ et al. Age at onset and Parkinson diseasephenotype. Neurology. 2016;86(15):1400-1407.

26. Suchowersky O, Reich S, Perlmutter J et al. Quality Standards Parameter: diagnosis and prognosis of new onset Parkinson disease (an evidence-based review): report of the Quality Standards Subcommittee of the American Academy of Neurology.Neurology. 2006; 66(7):968-975.

27. Aarsland D, Brønnick K, Alves $G$ et al. The spectrum of neuropsychiatric symptoms in patients with early untreated Parkinson's disease. J Neurol Neurosurg Psychiatry. 2009; 80(8):928-930

28. Santangelo G, Vitale $C$, Trojano $L$ et al. Relationship between apathy and cognitive dysfunctions in de novo untreated Parkinson's disease: a prospective longitudinal study. Eur J Neurol. 2015;22(2):253-260.

29. Trojano L, Papagno C. Cognitive and behavioral disorders in Parkinson's disease: an update. I: cognitive impairments. Neurol Sci. 2018;39(2):215-223.

30. Li S, Ou R, Yuan X et al. Executive dysfunctions and behavioral changes in early drug-naïve patients with Parkinson's disease. J Affect Disord. 2019;243:525-530.

31. Vilas D, Pont-Sunyer C, Tolosa E. Impulse control disorders in Parkinson's disease. ParkinsonismRelatDisord. 2012;18.Suppl 1:S80-84.

32. Vriend C, Pattij T, van der Werf YD et al. Depression and impulse control disorders in Parkinson's disease: two sides of the same coin? NeurosciBiobehav Rev. 2014;38:60-71.

33. Leentjens AF, Van den Akker M, Metsemakers JF et al. Higher incidence of depression preceding the onset of Parkinson's disease: A register study. Mov Disord. 2003;18(4):414-418. Subcommittee of the American Academy of Neurology. Practice 